\title{
Visualization of nonlinear kernel models in neuroimaging by sensitivity maps
}

\author{
Rasmussen, Peter Mondrup; Hansen, Lars Kai; Madsen, Kristoffer Hougaard
}

Publication date:

2010

Link back to DTU Orbit

Citation (APA):

Rasmussen, P. M., Hansen, L. K., \& Madsen, K. H. (2010). Visualization of nonlinear kernel models in neuroimaging by sensitivity maps. Abstract from 16th Annual Meeting of the Organization for Human Brain Mapping, Barcelona, Spain.

\section{General rights}

Copyright and moral rights for the publications made accessible in the public portal are retained by the authors and/or other copyright owners and it is a condition of accessing publications that users recognise and abide by the legal requirements associated with these rights.

- Users may download and print one copy of any publication from the public portal for the purpose of private study or research.

- You may not further distribute the material or use it for any profit-making activity or commercial gain

- You may freely distribute the URL identifying the publication in the public portal

If you believe that this document breaches copyright please contact us providing details, and we will remove access to the work immediately and investigate your claim. 


\section{Visualization of nonlinear kernel models in neuroimaging by sensitivity maps

\author{
P. M. Rasmussen $\dagger^{\star}$, K. H. Madsen‡, T. E. Lund ${ }^{\star}$, L. K. Hansen $\dagger$
} \\ $\dagger$ †TU Informatics, Technincal University of Denmark. $\ddagger$ Danish Research Center for Magnetic Resonance, Hvidovre, Denmark. Center of Functionally Integrative Neuroscience, Aarhus, Denmark.

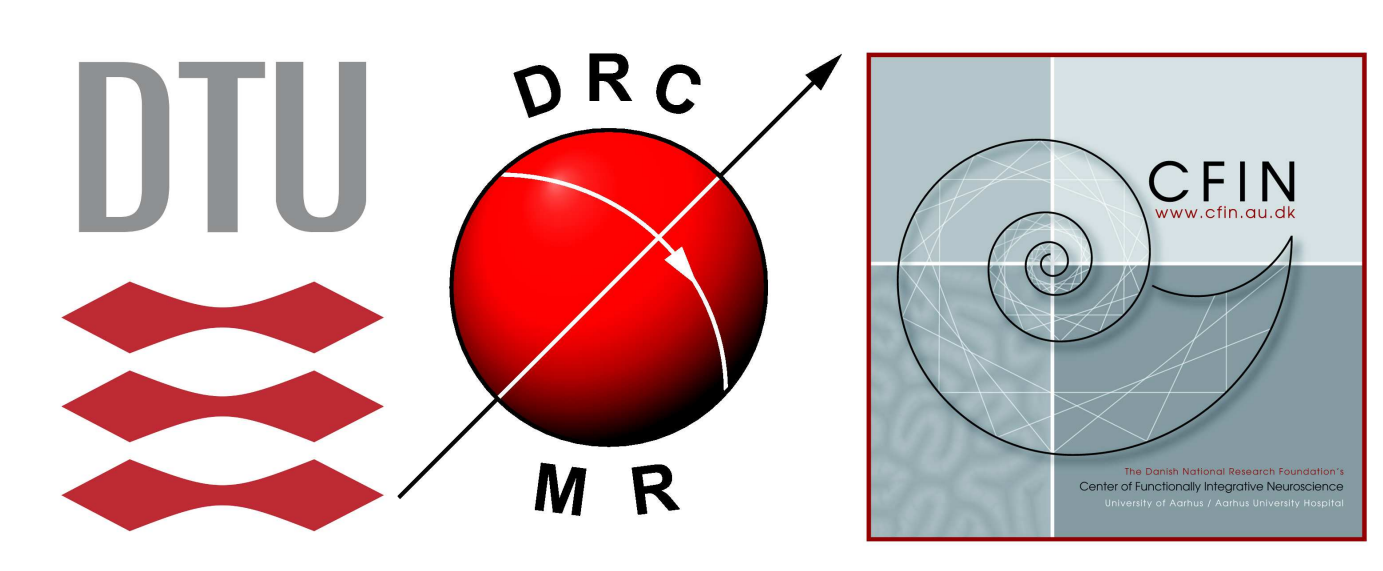

\section{Aim}

Recently there has been renewed interest in decoding information about mental states from distributed representational patterns in neuroimaging. It is highly desirable to identify in which brain locations the discriminative information resides. Here we investigate a model visualization for nonlinear kernel methods based on sensitivity analysis.

Simulation: XOR coupling.

\section{Materials and Methods}

XOR coupling.

fMRI: Finger tapping experiment. 8 subjects. Block design. Two conditions: left and right hand finger tapping.10 rep. of each condition per subject.

\section{Classification methods}

Support vector machine (SVM).

- Logistic regression (LogReg).

- Relevance vector machine (RVM).

Model evaluation

NPAIRS framework. Prediction accuracy and pattern reproducibility metrics.

\section{Conclusion}

Nonlinear classification models provide more flexibility than linear models. In addition to build a model with a good classification accuracy a competing goal is to interpret the model in terms of the neural representation that drives the predictions. The sensitivity map is a straightforward, yet powerful procedure for generation of meaningful global summary maps of nonlinear kernel methods.

\section{References}

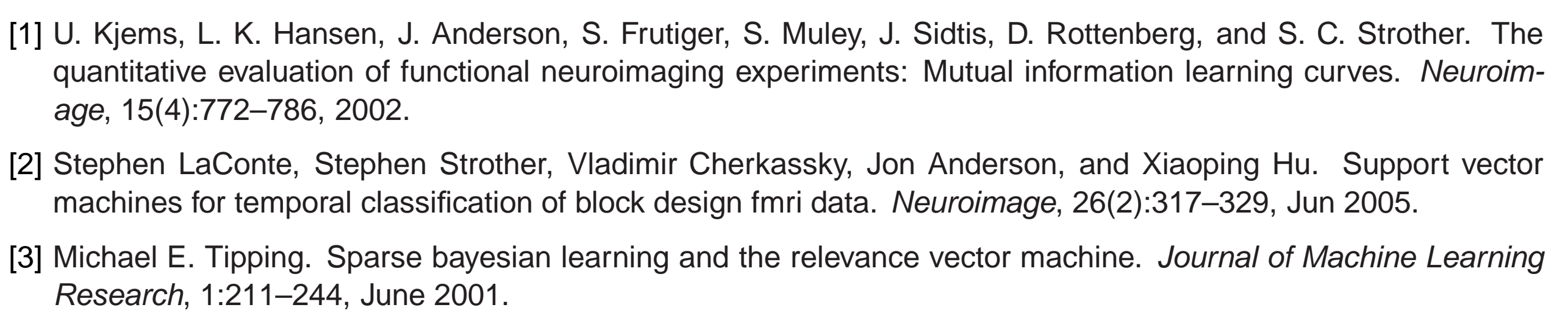

\section{Mathematical Modeling}

Consider a labeled data set $\mathcal{D}=\left\{\mathbf{x}_{n}, t_{n}\right\}_{n=1}^{N}$, where $\mathbf{x}$ is a $\mathrm{V}$ dimensional feature vector that, in the context of classification in fMRI, contains a brain scan volume or some derived features, while $t$ is the corresponding target variable. In a probabilistic framework the aim in supervised learning is to estimate the predictive distribution over $t$ for a new input $\mathbf{x}$

$$
p(t \mid \mathbf{x}, \mathcal{D})=\int p(t \mid \mathbf{x}, \mathbf{w}) p(\mathbf{w} \mid \mathbf{x}, \mathcal{D}) d \mathbf{w},
$$

where $\mathbf{w}$ are model parameters, while $p(t \mid \mathbf{x}, \mathbf{w})$ is the likelihood and $p(\mathbf{w} \mid \mathbf{x}, \mathcal{D})$ is the posterior distribution over the model parameters.

Binary classification: $p(t \mid \mathbf{x}, \mathbf{w})=\sigma\left(t \mathbf{w}^{\prime} \boldsymbol{\varphi}(\mathbf{x})\right)$

Linear model $y(\mathbf{x})=\mathbf{w}^{\prime} \boldsymbol{\varphi}(\mathbf{x})$, where basis functions $\varphi$ may be kernels.

$\mathbf{k}_{\mathbf{x}}$ holds the elements $k\left(\mathbf{x}_{n}, \mathbf{x}\right)$, where $n$ is the index of a specific training example. Examples are the linear kernel $k\left(\mathbf{x}_{n}, \mathbf{x}\right)=\mathbf{x}_{n}^{\prime} \mathbf{x}$, and the Gaussian kernel $k\left(\mathbf{x}_{n}, \mathbf{x}\right)=\exp \left(-\left\|\mathbf{x}_{n}-\mathbf{x}\right\|^{2} / 2 \sigma^{2}\right)$.

\section{Nonlinear classification}

Figure 1: Analysis of spatial brain patterns by using a multivariate approach. Each scan is represented in terms of a high dimensional pattern vector (here only shown two dimensions denoted by voxel A and voxe $B)$. In a classification scheme the goal is to assign the correct label/brain state to a new brain scan based on the spatial pattern vector. The symbols corresponds to two hypothetical brain states. In the middle pand the brain states are separable by a linear decision bound ary, while a nonlinear decision boundary is required in the example in the left panel.
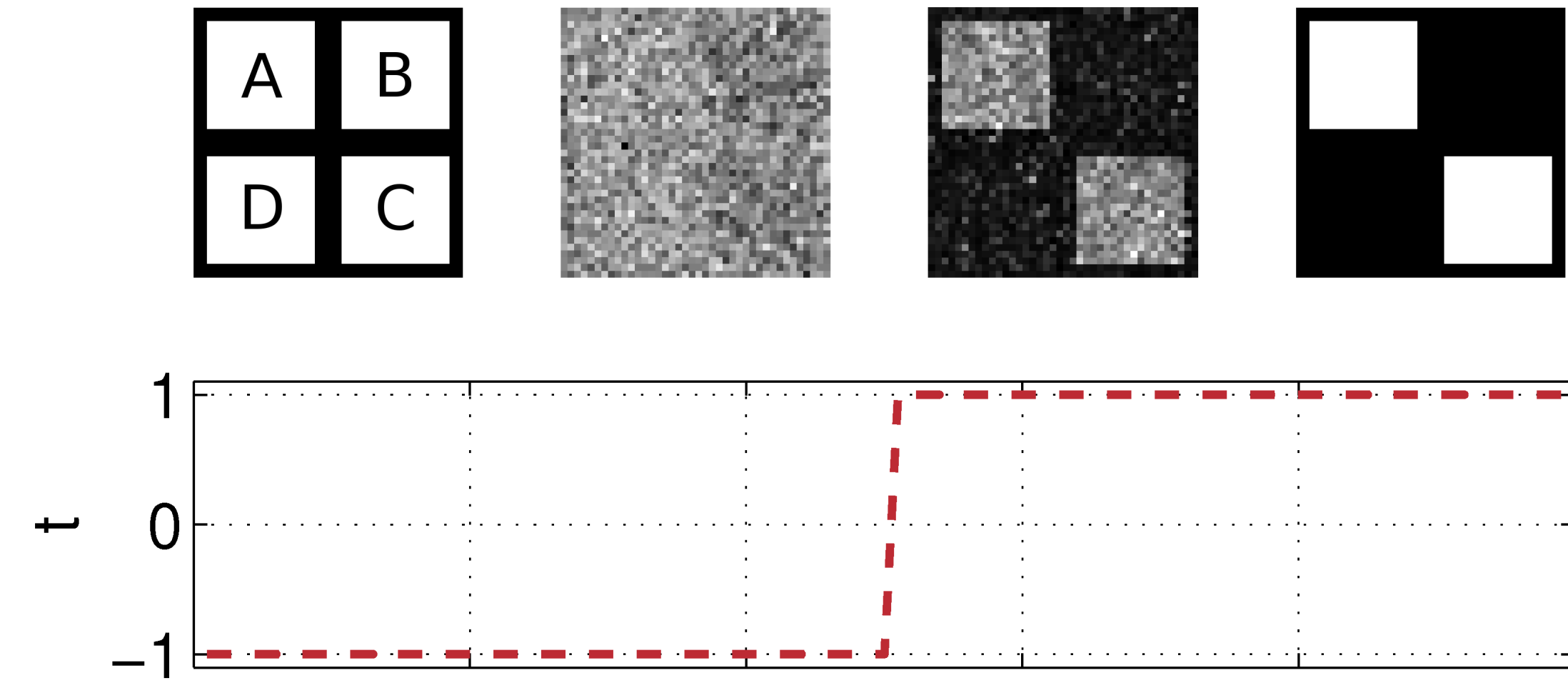

Fimulation of regions that interact through a nonlinear relationship. The interrelation between the two regions is important to the classification task. This configuration is aimed at modeling a set of brain regions in which the interaction is modulated by the brain state. There are four spatially contiguous regions that can activate denoted by $(A, B, C, D)$. Initially we let regions $(A, B, D)$ be activated by random sequence taking values \pm 1 . The target signal, coding for an initial baseline taking values $t_{n}=-1$, and for an active state $t=1$. The region $(C)$ is activated with an XOR-sequence relative to region $(\mathrm{A})$ and $t$, so that $C_{n}=A_{n} t_{\text {. }}$. The two regions $(B, D)$ are unrelated to the stimulus reference function $t_{n}$ but are included to ensure that the brain state can no be decoded based on regional measures, such as variance or non-Gaussianity. Additionally, Gaussian noise was added to the images to obtain a signal to noise ratio of 0.2 . Classification accuracy with an SVM was $90 \%$ while the corresponding model with a linear kernel achieved $50 \%$ accuracy (corresponding to random guessing). Top panel left to right simulation setup, "true map".

\section{Model visualization}

Usual visualization of linear kernel methods in functional neuroimaging

$$
y(\mathbf{x})=\mathbf{w}^{\prime} \mathbf{k}_{\mathbf{x}}=\mathbf{w}^{\prime} \mathbf{X} \mathbf{x}
$$

where training examples are ordered in the rows of $\mathbf{X} . \mathbf{w}^{\prime} \mathbf{X}$ is then considered as a discriminative map. We propose the sensitivity map as a general visualization of kernel methods in neuroimaging.

$$
s_{j}=\int\left(\frac{\partial \log p(t \mid \mathbf{x}, \mathcal{D})}{\partial x_{j}}\right)^{2} p(\mathbf{t}, \mathbf{x}) d \mathbf{t} d \mathbf{x},
$$

where the visualization $s_{j}$ resides in the same domain as the input data. In some models it is convenient to perform the integrals, in most cases we will approximate by empirical means over the training set $\mathcal{D}$. The sensitivity analysis or sensitivity map is a simple measurement of to what extent the predictive performance of a model depends on a given input.

For logistic regression

$$
s_{j}=\int \gamma(\mathbf{w}, \mathbf{x})\left(\frac{\partial \mathbf{w}^{\prime} \mathbf{k}_{\mathbf{x}}}{\partial x_{j}}\right)^{2} p(\mathbf{x}) d \mathbf{x}
$$

where $\gamma(\mathbf{w}, \mathbf{x})=\left(1-\sigma\left(\mathbf{w}^{\prime} \mathbf{k}_{\mathbf{x}}\right)\right) \sigma\left(\mathbf{w}^{\prime} \mathbf{k}_{\mathbf{x}}\right)$.

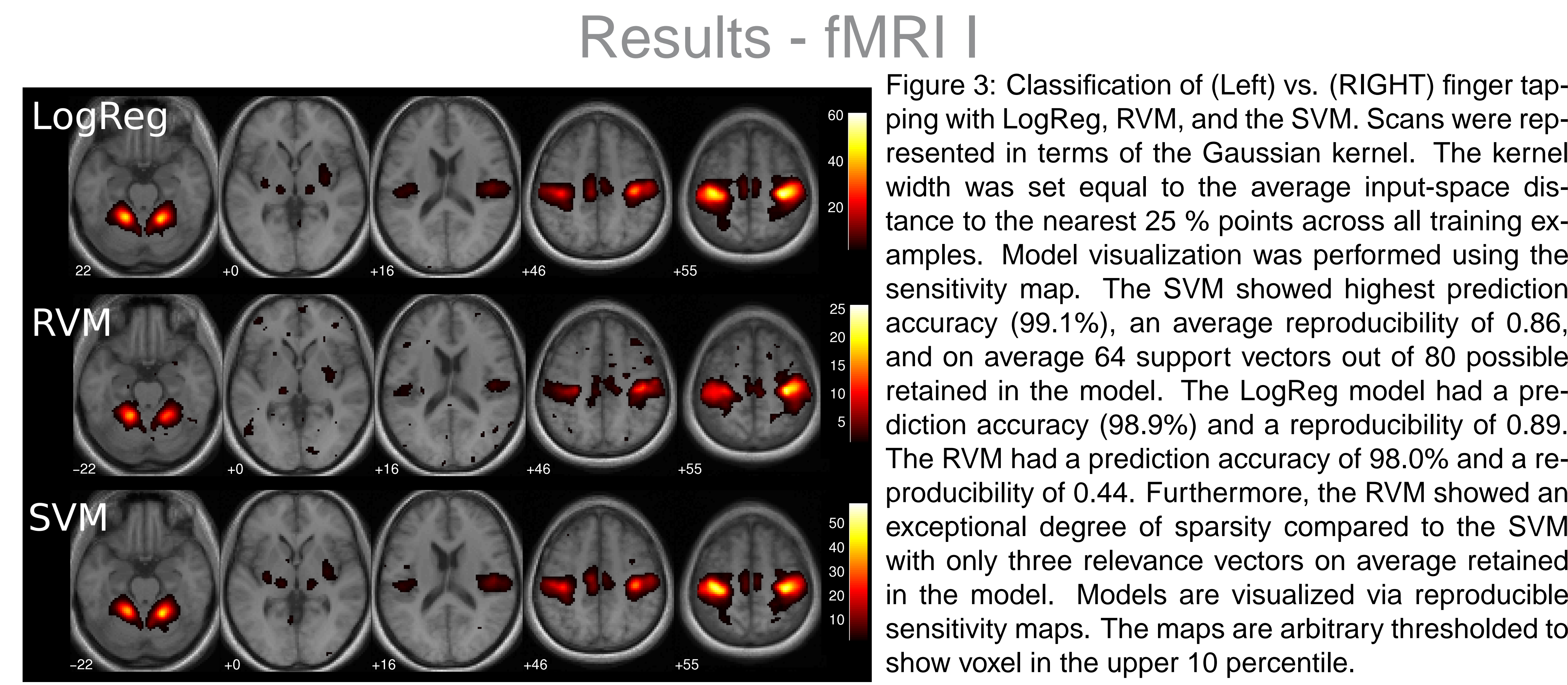

